\title{
Application Research of the Page Test in Active Sonar Detection
}

\author{
YOU Bo and CAI Zhi-ming
}

College of Electronic Engineering, Naval University of Engineering, Wuhan , 430033, China

Email: bbyouyou2002@yahoo.com.cn

\begin{abstract}
A feedback mechanism of the Page test is proposed in this paper. From the aspects of SNR estimation on square output of the matched filter, normalization, and the gate calculation, the information feedback flow is discussed. A new one-threshold Page test and processing flow that can effectively distinguish between target and interferences by cumulating change process adequately are also proposed. The performance of advanced Page test with feedback mechanism is improved evidently, especially to those signals with low target SNR and lower frequency. Data from the experiments on the sea proved the Page test behave well in actual oceanic conditions.
\end{abstract}

\section{Introduction}

The Page test designed for the detection of a change was originally brought forward by English scientist Page E.S. in 1950's. In recent 20 years it has been widely applied in many research fields such as signal processing, pattern recognizing, quality controlling and malfunction detection etc. In 2002 Douglas A. Abraham first applied the Page test to active sonar signal processing ${ }^{[1]}$.In 2006 the Page test was integrated into the sonar processing chain of the low frequency active systems (LFAS) in Italy NATO Undersea Research Center ${ }^{[2]}$.

The postpositional temporal signal processing is based on two fundamentals. One is the change of probability deviation function before and after target is present, which determines the concrete form of the test statistic. The other is the utilization of channel circumstance information. Signal power, belonging to one target, is cumulated according to SNR at square output of the matched filter whose precision affects the performance of the Page test. In practice, the SNR estimation and high false alarm numbers are the main problems. The building of feedback mechanism can improve the SNR estimation precision and reduce output fluctuation caused by stochastic interferences. The new one-threshold Page test and processing flow can distinguish between target and interferences by cumulating change process adequately. So it can suppress false alarms effectively. The data from the experiments on the sea proved the advanced algorithm behave well in actual oceanic conditions, especially to those signals with low target SNR and lower frequency.

\section{Feedback mechanism of the Page test}

The Page Test was designed to detect a change in the distribution of a sequence of data. It may be viewed as consecutive Wald sequential probability ratio tests with the test statistic written as

$$
\left\{\begin{array}{l}
W_{0}=0 \\
W_{n}=\max \left\{0, W_{n-1}+g(y[n])\right\}
\end{array}\right.
$$

where $g(y)$ is the detector nonlinearity. The Page test requires that the expected value of the detector nonlinearity be negative under the null or before change hypothesis $(\mathrm{H} 0)$ and positive under the alternative or after change hypothesis (H1) : $E\left[g \mid H_{0}\right] \leq 0<E\left[g \mid H_{1}\right]$. Before change, the test statistic $\mathrm{W}_{\mathrm{n}}$ equals to zero with greater probability and is hard to accumulate to cross the threshold. During the change lasting time, the test statistic $\mathrm{W}_{\mathrm{n}}$ is positive with greater probability and is easy to accumulate to cross the threshold.

Flow and feedback diagram of processing of array data is depicted in Fig.1. Automatic detectors are implemented after the matched filter squared output 
has been normalized ${ }^{[3]}$ by estimates of the reverberation and background noise power.

The left steps in Fig. 1 are realized by using the acoustic field calculation model to predict or initialize. They include using the ray methods to calculate the echoes spreading time as the reference of the guard band length in normalization, and using wave propagation theory to predict the transmission loss and the reverberation level[4] in the sonar equation so as to calculate the output SNR series of the matched filter. The initialization step includes designing $10 \mathrm{~dB}$ for the threshold calculation because it is the lowest SNR that the sonar operator can determine from noise background. The right steps in Fig.1 are feedbacks from processing results. The errors between actual ocean circumstances and prediction results from acoustic field calculation model are corrected by those steps. They include using the primary processing results of the Page test, such as the estimation of the starting and stopping times of the possible received echo to correct the prediction results of the echo spreading time, and using the actual SNR of the possible target to adjust the SNR series evaluated by sonar equation.

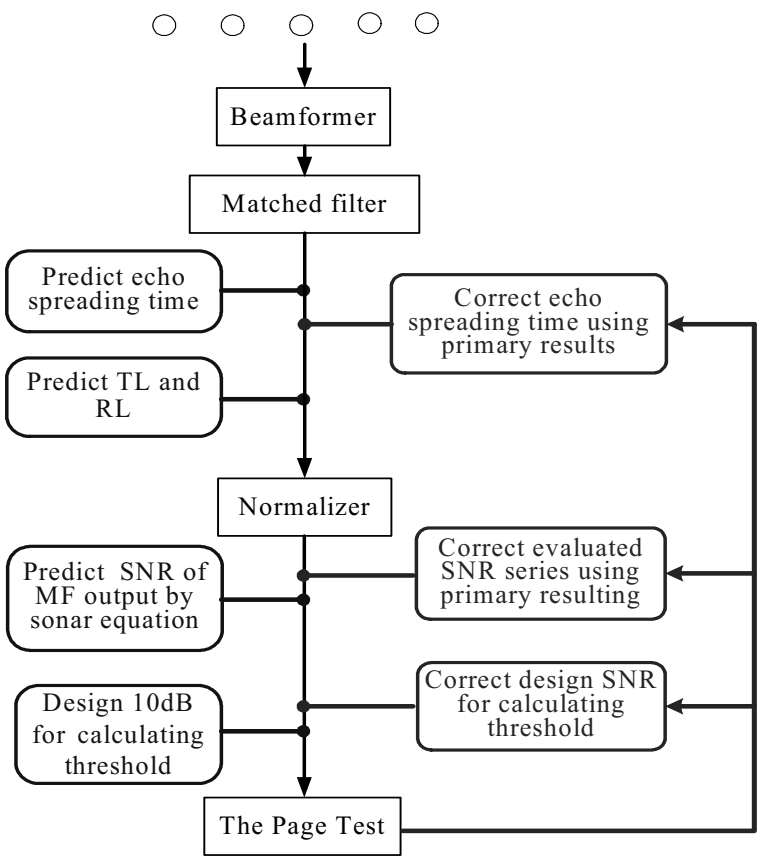

Fig1. Flow and feedback diagram of processing of array data before the Page test
The detection performance is directly influenced by the evaluated SNR series. We artificially adjust the target SNR of the active sonar echoes obtained from the experiment on the sea. We choose a HFM waveform with bandwidth $500 \mathrm{~Hz}$. The target SNR varies from $4.8 \mathrm{~dB}$ to $11.8 \mathrm{~dB}$. Under the condition that the detection performance keeps invariant, the upper and lower tolerance are calculated. The lower tolerance of SNR is $3 \mathrm{~dB}$. The upper tolerance varies with the target SNR as depicted in Fig.2. So the tolerable mismatch range increases with target SNR. When the target $\mathrm{SNR}$ is $11.8 \mathrm{~dB}$, the range can reach $77 \mathrm{~dB}$. When the target SNR equals to $4.8 \mathrm{~dB}$, the range is only $3 \mathrm{~dB}$. So it is concluded that the Page test is in good tolerance of the mismatch of evaluated SNR, only when the target SNR is high. To those signals with low target SNR, the precision of evaluated SNR series affects the detection performance evidently. The SNR precision evaluated by sonar equation is limited, while the building of feedback mechanism of the Page Test resolves this problem. As depicted in Fig.1, the evaluated SNR is corrected by the actual SNR of the possible target in the matched filter square output after primary detection.

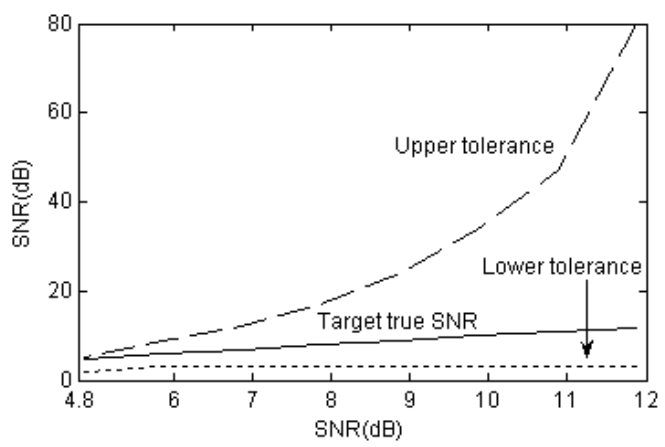

Fig.2 Analysis of the $S N R$ mismatch tolerance of the

\section{Page test}

\section{The new algorithm flow to suppress false-alarming interference}

Fig.3 describes the general algorithm flow of the SST-Page test ${ }^{[1]}$. The flow within dashed line frame needs to be modified. The threshold $\left(h_{0}\right)$, detector nonlinearity $\left(g_{n}^{0}\right)$ and the test statistic $W_{n}=\max \left\{0, W_{n-1}+g_{n}^{0}\right\}$ for the Page test searching for 
the onset of a signal are different from those $\left(h_{l}, g_{n}^{1}\right.$ and $\left.W_{n}=\min \left\{h_{0}+h_{1}, W_{n-1}+g_{n}^{l}\right\}\right)$ for the Page test searching for the termination of the signal. The starting time $n_{s}$ is the last zero point before $W_{n}$ will cross the threshold $h_{0}$. The stopping time $n_{e}$ is the last point equaling to $h_{0}+h_{1}$ before $W_{n}$ is lower than threshold $h_{0}$. From the former analysis it is concluded when $W_{n}$ crosses the threshold $h_{0}$, the test statistic will be set to $h_{0}+h_{1}$ no matter it is the true target or any false alarm interference. This will increase the false alarm numbers actually.

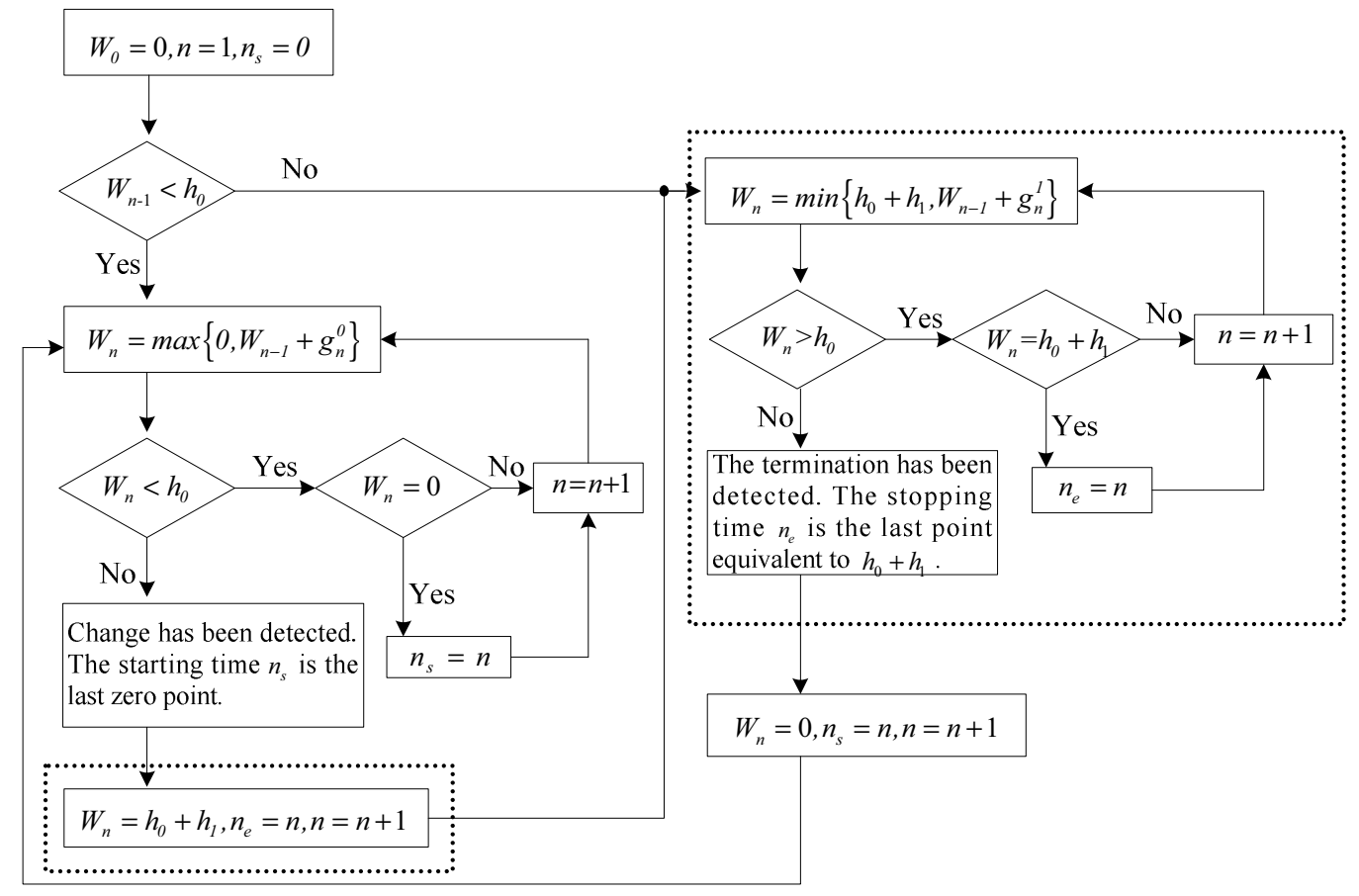

Fig.3 The general algorithm flow of the SST-Page test

The advanced algorithm flow not only simplifies the double-threshold design to the new one-threshold design, but also changes the test statistic to be $W_{n}=W_{n-1}+g_{n}^{l}$ when $W_{n}$ crosses the threshold $h_{0}$. When the change happens, the change process will be cumulated adequately to distinguish true target strength from false alarm strength. The foundation of doing so is the inequation $E\left[g \mid H_{0}\right] \leq 0<E\left[g \mid H_{1}\right]$ which the detector nonlinearity must meet as mentioned before. The cumulating process gives prominence to the target strength and suppresses false alarm interferences. When the Page test searches for the termination of the signal, we suppose the point at which $W_{n}$ is lower than threshold $h_{0}$ as $n_{e}^{\prime}$. Then the stopping time $n_{e}$ from which $W_{n}$ finally begins to descend is the last maximum point in the range from the starting time $n_{s}$ to $n_{e}^{\prime}$. In fact, this judgment equals to the former one that designs that the stopping time $n_{e}$ is the last point equaling to $h_{0}+h_{1}$ before $W_{n}$ is lower than threshold $h_{0}$.The advanced algorithm simplifies the flow and avoids exorbitant computation.
After confirming the value of $n_{s}$ and $n_{e}$, we design $W_{n s: n e}$ to be the maximum between $n_{s}$ and $n_{e}$ to emphasize the target location further, which is $W_{n s: n e}=\max \left(W_{n s}: W_{n e}\right)$.

We add three transient changes to a Gaussian stochastic sequence with mean $\mu=2$ and variance $\sigma^{2}=1$. The first change is between $200^{\text {th }}$ and $210^{\text {th }}$ point with mean $\mu^{\prime}=2$. The second is between the $300^{\text {th }}$ and the $330^{\text {th }}$ point with $\mu^{\prime}=2.6$. The third is between the $400^{\text {th }}$ and the $405^{\text {th }}$ with $\mu^{\prime}=2$. According to the choosing principle ${ }^{[5]}$ of nonlinearity $g(y)$ and transformation of probability deviation function before and after transient change, we get $g(y)=y-b$ with bias $b=\left(\mu+\mu^{\prime}\right) / 2$. The comparison between the double-threshold Page test result and the one-threshold result is depicted in Fig.4. The results of starting time of two methods are consistent. In the output series of advanced algorithm, we can distinguish clearly strengths of transient changes with different lasting time and mean. However the former algorithm outputs of different 
changes are limited to the same value and it is unable to distinguish different changes. So in practical sonar signal processing, the advanced algorithm can suppress those false alarm interferences including those with short duration in underwater acoustic channels or sonar circuits.
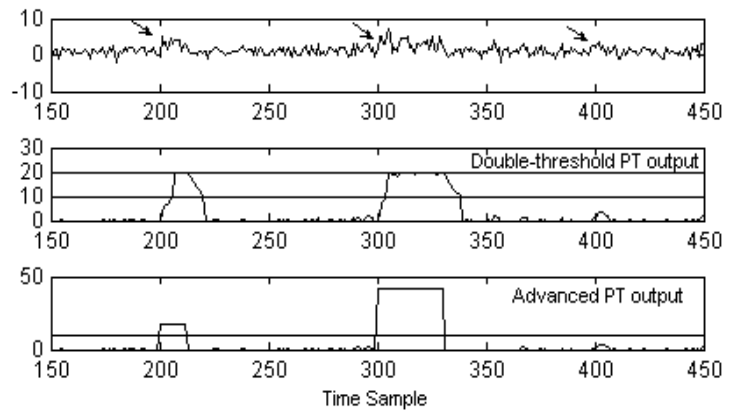

Fig.4 Output comparison between the Page test and the

\section{Real data example}

The environmental parameters obtained from the practical experiment in certain sea area are illustrated in Table 1. The source depth was $10 \mathrm{~m}$ in isovelocity water. The active sonar eradiated HFM signals with bandwidth $\mathrm{B}=500 \mathrm{~Hz}$. The reverberation power level estimated by the normalizer is adjusted by incoherent reverberation power which was estimated by normal mode acoustic propagation model. A source level was utilized as $210 \mathrm{~dB}$ and target strength as $8 \mathrm{~dB}$. Using the ray method, as the reference of the guard band length in normalization, the channel spreading time is about $0.06 \mathrm{~s}$.

Table 1 parameters of the environment

\begin{tabular}{|c|c|c|c|c|}
\hline $\begin{array}{c}\text { Depth } \\
(\mathrm{m})\end{array}$ & $\begin{array}{c}\text { Sound } \\
\text { velocity } \\
(\mathrm{m} / \mathrm{s})\end{array}$ & $\begin{array}{c}\text { Seabed Sound } \\
\text { velocity }(\mathrm{m} / \mathrm{s})\end{array}$ & $\begin{array}{c}\text { Seabed } \\
\text { density } \\
(\mathrm{g} / \mathrm{cm} 3)\end{array}$ & $\begin{array}{c}\text { Seabed } \\
\text { wave } \\
\text { absorption } \\
\mathrm{dB} / \lambda\end{array}$ \\
\hline 70 & 1520 & 1709 & 1.88 & 0.5 \\
\hline
\end{tabular}

Results of temporal and spatial normalization and advanced Page test with feedback mechanism are compared in Fig.5. Both methods can smooth reverberation effectively. The background of the latter is cleaner and the target is more prominent than the former. To the signal with lower frequency and lower SNR, though the general normalization algorithm can't distinguish target from background, the advanced Page test can distinguish target clearly. In conclusion, the performance of the advanced Page test with feedback mechanism is improved evidently, and it can adapt well to the background changes of sonar receiver and the actual oceanic condition.
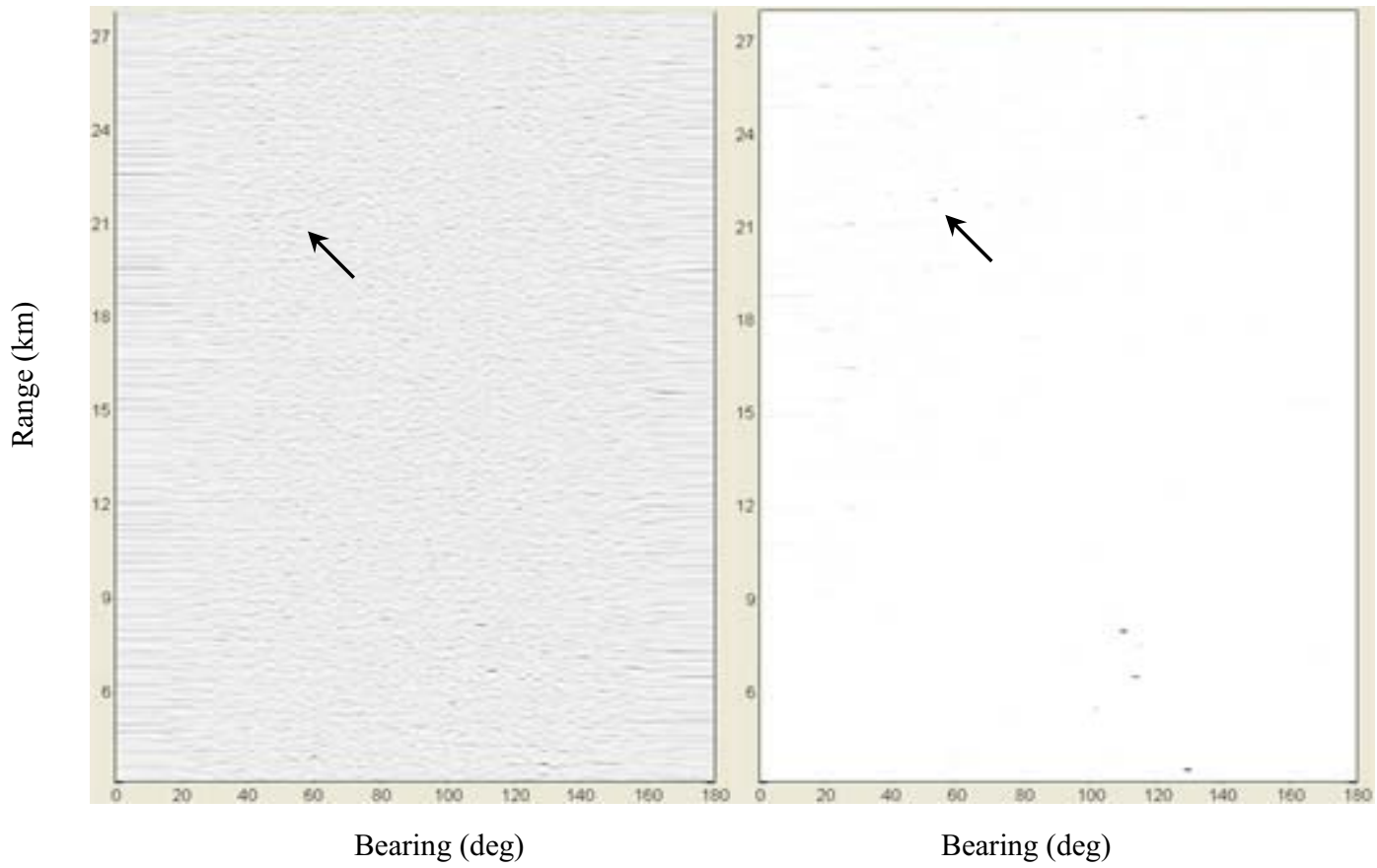

Fig.5 Output comparison between general normalization and advanced Page test with feedback mechanism 


\section{Conclusion}

On one side, a feedback mechanism of the Page test is proposed in this paper. From the aspects of SNR estimation on square output of the matched filter, normalization, and the gate calculation, we discussed the information feedback flow. We also studied the mismatch effect of estimated SNR series on the detection performance. The tolerable mismatch range increased with target SNR. The performance of Page test with feedback mechanism was improved evidently, especially to those signals with low target SNR. On the other side, a new one-threshold Page test and new processing flow are also proposed in this paper. The new method could effectively distinguish between target and interferences by cumulating change process adequately. All those amendments made the Page test behave well in actual oceanic conditions.

\section{References}

[1] Douglas A. Abraham, Peter K.Willett. Active Sonar Detection in Shallow Water Using the Page Test [ J ]. IEEE Trans. Aerosp. Electron. Syst., 27(1): 1225-1229, 2002.

[2] Alberto Baldacci, Georgios Haralabus . Signal processing for an active sonar system suitable for advanced sensor technology applications and environmental adaptation schemes. SACLANT Undersea Research Centre, La Spezia,Italy,NURC-PR-019, 2006.

[3] Baldacci, A. , Haralabus, G. , Adaptive normalization of active sonar data. SACLANT Undersea Research Centre, La Spezia, Italy, Report PR-013, 2006.

[4] Dale D.Ellis, A shallow-water normal-mode reverberation model $[\mathrm{J}]$. J.Acoust. Soc. Am., 97(5): 2804-2814, Pt.1, May 1995.

[5] Douglas.A.Abraham. Asymptotically optimal bias for a general nonlinearity in Page's Test[J]. IEEE Trans. Aerosp and Electron Syst, Jan. 32:360-367, 1996. 